\title{
Critical roles of TLRs on the polarization of mesenchymal stem cells for cell therapy of viral infections: a notice for COVID-19 treatment
}

\author{
Shiva Gholizadeh-Ghaleh Aziz ${ }^{1}$ - Shahriar Alipour ${ }^{1}$ • Parviz Ranjbarvan ${ }^{1} \cdot$ Arezo Azari $^{2}$ • Ghader Babaei ${ }^{1}$. \\ Ali Golchin ${ }^{1,3}$ (D)
}

Received: 23 October 2020 / Accepted: 24 January 2021 / Published online: 29 January 2021

(C) The Author(s), under exclusive licence to Springer-Verlag London Ltd. part of Springer Nature 2021

\begin{abstract}
Mesenchymal stem cells (MSCs), as one of the leading cell-based therapy, have provided a strong link between clinical investigation and basic research. MSCs have been successfully employed in treating graft versus host disease (GvHD), autoimmune disease, and several other diseases, particularly with high immune activity. Recently, MSCs have attracted attention to treating untreatable viral infections such as severe coronavirus disease 2019 (COVID-19). Given that the Toll-like receptors (TLRs) are directly able to detect internal and external hazard signals, and their stimulation has an intense effect on the ability to grow, differentiate, migrate, and maintain MSCs, it seems stimulation of these receptors can have a direct impact on the interaction of MSCs and immune cells, altering the ability to modify immune system responses. Hence, this mini-review focused on TLRs' critical roles in the polarization of MSCs for developing MSC-based therapy in viral infections. Consequently, according to the literature review, a polarization process, mediated by TLRs concerning anti-inflammatory and proinflammatory phenotype, may be considered for MSC-therapy against viral infections.
\end{abstract}

Keywords Mesenchymal stem cells $\cdot$ Toll-like receptors $\cdot$ Viral infections $\cdot$ Anti-inflammatory agents $\cdot$ COVID-19

\section{Introduction}

Over the past decade, extensive research of the immunemodulatory and tissue regeneration potency of mesenchymal stem cells (MSCs) has demonstrated the valuable promise of these cells to prevent and treat immune-related disorders and even viral diseases. The diverse origin of MSCs, based on their immunomodulatory and regenerative potency, has attracted attention in the cellular-based therapy field (Golchin et al. 2018, 2020c). In biological systems, MSCs render beneficial effects, such as supporting the proliferation and function of antiviral distinct effector cells, promoting healthy physiology against

Ali Golchin

agolchin.vet10@yahoo.com; Golchin.a@umsu.ac.ir

1 Department of Clinical Biochemistry and Applied Cell Sciences, Faculty of Medicine, Urmia University of Medical Sciences, Urmia, Iran

2 Department of Tissue engineering and Applied Cell Sciences, School of Advanced Technologies in Medicine, Shahid Beheshti University of Medical Sciences, Tehran, Iran

3 Regenerative Medicine Group (REMED), Universal Scientific Education and Research Network (USERN), Tehran, Iran viral hurdle by the antimicrobial activity, and generating angiogenic growth factors (Golchin et al. 2020a). MSCs can be extracted from various embryonic and non-embryonic sources and include a total heterogeneous population of progenitor cells whose unique innate abilities can be expected in their therapeutic usage (Akira and Sato 2003; Judge et al. 2005; Hwa Cho et al. 2006; El Omar et al. 2014). One of the properties of bone marrow-derived MSCs are the expression of a wide range of Toll-like receptors (TLRs) such as TLRs 1, 2, 3, 4, 5, 6, 8, 9, and 10 (Li et al. 2014), the Wharton jelly, and umbilical cord blood-displayed TLRs 1, 2, 3, 5, 6, and 9 (Berk et al. 2010; Raicevic et al. 2011), various expression in the periodontal ligament (Li et al. 2014), dental follicle and dental pulp (Tomic et al. 2011), and free gingiva (Fawzy-El-Sayed et al. 2016; Mekhemar et al. 2018). The study of Toll-like receptor (TLRs) in different MSCs isolated from diverse sources, such as human umbilical cord blood-derived MSCs (hUCB-MSCs), human adipose-derived MSCs (hAT-MSCs), human dental pulp-derived MSCs (hDP-MSCs), and human dental folliclederived MSCs (hDF-MSCs)), reveal the pattern of expression of TLRs that is closely associated with the tissue origin isolated from them (Hwa Cho et al. 2006; Raicevic et al. 2011). Recently, MSCs have been proposed as a possible therapeutic 
for COVID-19 treatment (Golchin et al. 2020b; Golchin 2020). MSCs display a significant shift in the proinflammatory pulmonary microenvironment induced by different conditions such as specific intoxications, ventilator-induced trauma, and infectious lung injury models (Monguió-Tortajada et al. 2020). MSCs demonstrated significant roles in the immune systems immunomodulatory process of severe COVID-19 patients. Based on reported MSC-based clinical studies in COVID-19 patients, MSC infusion causes which lymphocyte subpopulation (NK cells, B cells, CD4+ T cells, and CD8+ T cells) count and IL10 release are increased (Sánchez-Guijo et al. 2020), and concurrently overactivated cytokine-secreting cell count, Creactive protein, TNF- $\alpha$, and IL6 levels are decreased (Golchin 2020). We reviewed the proinflammatory and immunomodulatory effects of MSCs in COVID-19 patients in our recent studies (Golchin et al. 2020b; Golchin 2020). However, the role of TLR signaling in the therapeutic targets of MSCs should be considered (Monguió-Tortajada et al. 2020). The immunomodulation quality of MSCs has been linked to TLR receptors' expression and stimulated by pathogen-associated particles like LPS of infectious agents such as SARS-CoV-2 (Monguió-Tortajada et al. 2020).

Biological behavior and therapeutic effects of MSCs derived from different sources have been investigated for several viral infection diseases (Table 1) in preclinical and clinical studies. Interleukin (IL) 6 and10, transforming growth factor (TGF)- $\alpha$, indoleamine 2,3-dioxygenase (IDO), and MSC immunomodulatory activities can mediate prostaglandin E2. Virus-associated diseases have been assessed by the therapeutic potency of MSCs or their derived products. Currently, MSC-based therapy has become an encouraging choice for the possible treatment of new and hazardous diseases such as Coronavirus disease 2019 (COVID-19), immunologic abnormality in human immunodeficiency virus (HIV), acute lung injury (ALI) in the influenza virus, and chronic hepatitis in hepatitis B virus (HBV). Several countries, such as China, USA, Iran, and Spain, have recently begun clinical MSC-based therapy studies in patients of COVID-19 (Golchin et al. 2020b). Literature reviews have shown several significant results related to MSC behavior and viral infections, including the following (Ahmed Al-Anazi et al. 2020):

(1) MSCs are liable to be influenced by members of the herpes viruses group such as herpes simplex virus (HSV) type 1, HSV-2, cytomegalovirus, Epstein-Barr virus, and varicella-zoster virus (VZV), and subsequently become functionally defective.

(2) Some of the exceptions confirm the modified behavior of MSCs in related viral infected cells; for instance, ATMSCs undergoing hepatic cell differentiation are no sensitive to exposure of the HBV in vitro.

(3) Human MSCs are more sensitive to the particularly pathogenic avian influenza infection like $\mathrm{A} / \mathrm{H} 5 \mathrm{~N} 1$, and their infection can influence their viability by inducing apoptosis and losing their immunomodulatory potency.

(4) MSCs can notably decrease the pathogenic signs of alveolar fluid clearance created by influenza $\mathrm{A} / \mathrm{H} 5 \mathrm{~N} 1$ infection cell culture condition and also prohibit or lessen influenza $\mathrm{A} / \mathrm{H} 5 \mathrm{~N} 1$-associated acute lung injury in patients (Maytawan Thanunchai 2015; Chan et al. 2016; Al-Anazi* et al. 2019).

This mini-review is aimed at gathering and discussing TLR-related aspects of MSC therapy in inflammation and, subsequently, viral infections. Hence, given that the TLRs are directly able to detect several crucial signals, and their stimulation has an intense effect on their ability to proliferate, differentiate, migrate, and survive, it seems plausible that stimulation of these receptors has a direct impact on the interaction of MSCs with immune cells, altering the ability to modify immune system responses. Therefore, this study has focused on TLRs' critical roles in the polarization of MSCs for developing MSC-based therapy in viral infections.

\section{TLRs and MSCs in inflammation}

TLRs detect molecular patterns associated with microbes (MAMPs), and each TLR identifies a specific type of these molecular patterns. For example, TLR2 detects peptidoglycans, and TLR4 identifies lipopolysaccharide. TLRs are expressed by many immune cells, including dendritic cells, macrophages, neutrophils, and B and T cells. Activation of TLRs induces MHC class II molecules (the first signal) and helps stimuli such as CD80, CD86, and CD40, which provide the second signal to activate $\mathrm{T}$ cells. The third signal is provided for T cells' activation by peripheral cytokines, strongly influenced by the type and amount of TLR (Goulopoulou et al. 2016). Activation of TLR4, for example, causes T cells to differentiate into the Th1 class by inducing IL-12 production, while activated TLR2 triggers the Th2-based immune response by producing IL-10 IL-13. Of the ten known types, TLR2 and TLR4 have been studied most extensively (Liu et al. 2013; Rogier et al. 2015). For instance, studies demonstrated a remarkable decrease in IL-17 and IL- $1 \beta$ production by splenocytes upon TLR2 and TLR4 stimulation, recommending abolishing Th17 differentiation (AbdollahiRoodsaz et al. 2008; Rogier et al. 2015).

Based on literature reviews, there is a considerable link between stimulation of specific TLRs and immune system modulating responses in MSCs (Tomchuck et al. 2008). Following the activation of TLRs by alarm signals, their activation leads to systemic and cellular responses; while following higher tissue pathogenesis, large amounts of trigger TLRs are released. Danger signals are either exogenously generated by infectious agents or endogenously by circulating risk 


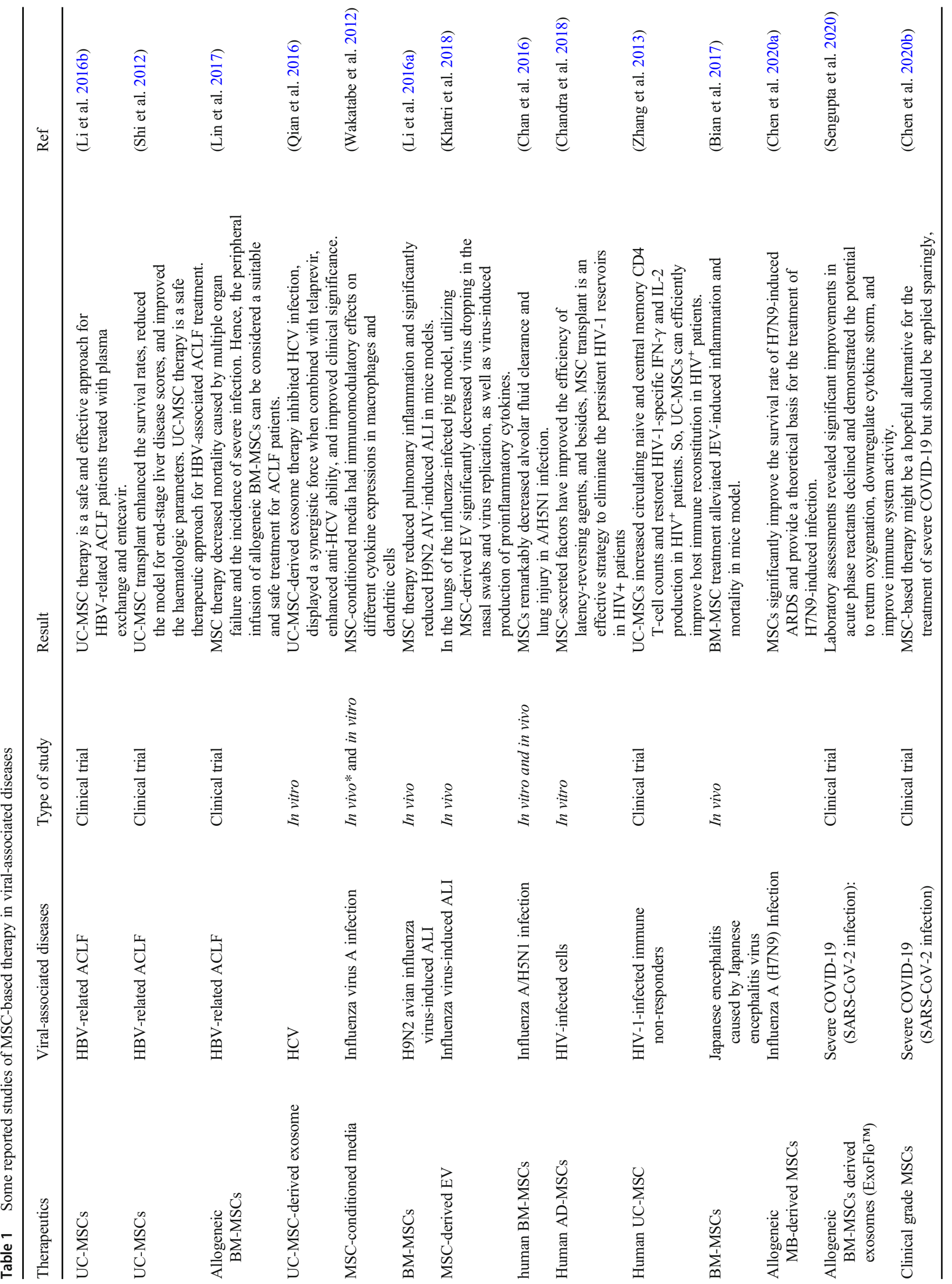


factors such as thermal shock proteins or RNA types (Goulopoulou et al. 2016). These signals activate the appropriate TLR to cause homeostasis in the body (Akira and Sato 2003; West et al. 2006). It has been shown that following the creation of alarm signals, immune cells are usually recruited into this area; thus, MSCs can exhibit similar mechanisms in finding these signals as immune cells. Also, MSCs not only have been shown to express several types of TLR but these cells also can migrate, attack, and secrete immune-modulating factors that interact with specific TLRs (Tomchuck et al. 2008). For example, a study found that stimulation of TLR3 secreted immune-inhibiting factors, while stimulation of TLR4 with lipopolysaccharides led to the secretion of inflammatory stimulants (Waterman et al. 2010). Other research has evaluated the effect of different TLRs on mesenchymal stem cells' characteristics in three layers (chondrogenic, fat, and bone). However, unlike human MSCs, they suggest that activating TLR2 leads to differentiation and migration of mouse MSC cells and increases their proliferation (Liotta et al. 2008). Conversely, another study found that activating TLRs did not affect the differentiation of human MSC in adipose tissue, cartilage, and bone (Liotta et al. 2008). Recent results from a survey by Lombardo et al. Found that the combination of TLR3 and TLR4 (TLR3 and TLR4 engagement) within hADSC cells increased bone differentiation values but did not affect the differentiation and proliferation of adipose tissue. The study also found that TLR2, 3, 4 ligations did not affect the ability of hADSC to inhibit lymphocyte activation (Lombardo et al. 2009).

According to studies, the mechanism for regulating the immune system seems to be complicated. The regulation of the immune system depends not only on the secretion of soluble factors but also on the communication and contact of MSCs with the immune system. Several factors have been linked to mesenchymal stem cells' immunosuppression, including IL-10, HLA-G, prostaglandins, interferon-gamma, and the Notch family as a low-signal signal to TLR associated with MSC (Nicola et al. 2002; Rizzo et al. 2008). However, there have been many studies and reports on the antagonistic and heterogeneous effects of the immune system on TLR activation and its role in stem cells. While understanding that TLRs are expressed in all cells among different species (mice and humans), similar responses are not always seen. Other causes that can lead to conflicting reactions in TLR responses in stem cells include the concentration and length of incubation with specific TLR agonists (Heinz et al. 2003; Mestas and Hughes 2004).

\section{TLRs and MSC interactions in viral infections}

Literature reviews demonstrate that specific TLRs' stimulation affects the immune-modulatory potency of hMSCs (Waterman et al. 2010; Rosa et al. 2012; Najar et al. 2017). 
As mentioned, TLRs recognize specific signals, and their activation leads to effective immune responses that can be mobilized by different host cells (Waterman et al. 2010). With regard to hMSCs expressing several TLRs (such as TLR3 and TLR4)(Waterman et al. 2010), so it seems this subject can be considered in the therapeutic application of MSCs in viral infectious diseases. Liu et al. demonstrated that the conditioned medium of hUC-MSCs inhibited the TLR4/NF-KB signaling pathway at both cells and living organisms (Liu et al. 2018). They also showed that inflammatory molecules' releasing and inflammatory cell infiltrating are decreased in unilateral ureteral obstruction (UUO)-induced renal fibrosis (Liu et al. 2018). Recently, Xu et al. studied the therapeutic effects of the combination of hUC-MSCs and liraglutide on liver injury and their mechanisms in rat models (Xu et al. 2020). They reported the positive effect of these therapeutics in liver injury, in which the mechanism was correlated to the TLR4/ NF-KB inflammatory pathway and oxidative stress. Therefore, increasing the expression of inflammatory cytokines alongside TLR4 and NF- $\mathrm{KB}$ in liver and serum was considerably restrained concurrently using hUC-MSCs and liraglutide (Xu et al. 2020). Wang et al. also reported a new paradigm for hMSCs related to TLRs signals that suggested that MSC polarization provides a suitable way to present these heterogeneous preparations of cells more uniformly and provide a functional aspect to consider improving current MSC-based therapies (Waterman et al. 2010). To date, 11 human (TLR1-TLR11) and 13 mouse (TLR1-TLR13) TLRs have been identified to recognize different microbial products from other microorganisms such as bacteria, viruses, and protozoa (Table 2).TLR family members were demonstrated in different cells and even different cell organelles (Table 2). For instance, TLR3, 7,8 , and 9 are displayed on the surface of lysosomes, endoplasmic reticulum, and endosomes identifying viral nucleic acids (Najar et al. 2017). TLR-related MSC signaling can be influenced by the physiopathological condition. For example, hypoxia distinctly heightened the mRNA level of TLR1, 2, 5, 9, and 10 (Cho

Table 2 Type of TLRs and their applications and functions

\begin{tabular}{|c|c|c|c|}
\hline TLRs & Source & Cell location & $\begin{array}{l}\text { Function in Microorganism } \\
\text { (Such Viruses) }\end{array}$ \\
\hline TLR1 & Monocytes and DCs & Cell surface & $\begin{array}{l}\text { Recognize microbial membrane } \\
\text { components: lipids, lipoproteins, } \\
\text { and proteins }\end{array}$ \\
\hline TLR2 & Macrophages and DCs & Cell surface & $\begin{array}{l}\text { Recognize microbial membrane } \\
\text { components: lipids, lipoproteins, } \\
\text { and proteins }\end{array}$ \\
\hline TLR3 & $\begin{array}{l}\text { Dendritic cells, CD8+ T cells, } \\
\text { NK cells, neurons, oligodendrocytes, } \\
\text { astrocytes, and microglia }\end{array}$ & $\begin{array}{l}\text { Intracellular compartments } \\
\text { (endoplasmic reticulum, } \\
\text { lysosomes, and endosomes) }\end{array}$ & Recognize viral nucleic acids \\
\hline TLR4 & $\begin{array}{l}\text { Innate immune cells, astrocytes and } \\
\text { microglia, bronchial epithelial } \\
\text { cells and alveolar cells }\end{array}$ & Cell surface & $\begin{array}{l}\text { 1: Recognize microbial membrane } \\
\text { components: lipids, lipoproteins, } \\
\text { and proteins } \\
\text { 2: Involved in recognizing molecular } \\
\text { patterns from SARS-CoV-2 }\end{array}$ \\
\hline TLR5 & $\begin{array}{l}\text { Alveolar macrophages, epithelial } \\
\text { cells of mucosal surfaces of the lung }\end{array}$ & Cell surface & $\begin{array}{l}\text { Recognize microbial membrane } \\
\text { components: lipids, lipoproteins, } \\
\text { and proteins }\end{array}$ \\
\hline TLR6 & $\begin{array}{l}\text { Dendritic cells, monocytes, } \\
\text { macrophages, microglia, neutrophils, } \\
\text { NK cells, B lymphocytes, appendix, } \\
\text { spleen, and lymph node }\end{array}$ & Cell surface & $\begin{array}{l}\text { Recognize microbial membrane } \\
\text { components: lipids, lipoproteins, } \\
\text { and proteins }\end{array}$ \\
\hline TLR7 & Plasmacytoid pre-dendritic cells & $\begin{array}{l}\text { Intracellular compartments } \\
\text { (endoplasmic reticulum, } \\
\text { lysosomes, and endosomes) }\end{array}$ & Recognize viral nucleic acids \\
\hline TLR8 & $\begin{array}{l}\text { Monocytes, macrophages, mast cells, } \\
\text { DCs, and microglia }\end{array}$ & $\begin{array}{l}\text { Intracellular compartments } \\
\text { (endoplasmic reticulum, } \\
\text { lysosomes, and endosomes) }\end{array}$ & Recognize viral nucleic acids \\
\hline TLR9 & Plasmacytoid pre-dendritic cells & $\begin{array}{l}\text { Intracellular compartments } \\
\text { (endoplasmic reticulum, } \\
\text { lysosomes, and endosomes) }\end{array}$ & Recognize viral nucleic acids \\
\hline TLR10 & $\begin{array}{l}\text { Spleen, lymph node, thymus, tonsil, } \\
\text { lung, dendritic cells, eosinophil, } \\
\text { neutrophils, and trophoblasts }\end{array}$ & Cell surface & $\begin{array}{l}\text { Recognize microbial membrane } \\
\text { components: lipids, lipoproteins, } \\
\text { and proteins }\end{array}$ \\
\hline
\end{tabular}


et al. 2010) while infectious MSCs with baculoviral vectors upregulated and stimulated the TLR3 signaling pathway(Chen et al. 2009; Najar et al. 2017). Based on some evidence, BM-MSCs can act as antigen-presenting cells and present viral antigens to cytotoxic T (CT) cells, although they are no wholly able to process the viral antigens (Nemeth et al. 2010). Liotta et al. reported that when BM-MSCs express the TLR3 and TLR4, their immune-suppressive response is obstructed, and the $\mathrm{T}$ cell response to double-stranded RNA viruses is recovered (Liotta et al. 2008). A considerable amount of literature demonstrates that TLRs are activated by viral RNA (e.g., TLR3) (as in SARS-CoV-2) and viral unmethylated CpG-DNA (e.g., TLR9), leading to downstream cell signaling pathways resulting in MSC activation (Waterman et al. 2010; Khoury et al. 2020). Hence, the interaction between MSCs behavior and viral infection is like a double-edged sword, but according to different studies, a polarization process, mediated by TLRs, about both antiinflammatory and pro-inflammatory phenotype can be considered for MSC-therapy against viral infections.

\section{Polarization of mesenchymal stem cells to MSC1 proinflammatory cells and their clinical applications}

TLRs are naturally able to detect internal and external hazard signals (Fig. 1). Their stimulation profoundly affects the ability of MSCs to grow, differentiate, migrate, and survive MSCs (Chen et al. 2009). Stimulation of these receptors can directly affect the interaction of MSCs and immune cells, altering the ability to modify immune system responses. Experimental evidence suggests that the pattern of expression and function of TLRs in MSCs is changed under different conditions and specific physiological stimuli. For example, exposure of MSCs to inflammatory agents' environments affects TLRs' expression and function in these cells. Also, between the interactions of MSCs with bacterial components through stimulation of TLRs, they can change the immune modulation caused by MSCs (Pevsner-Fischer et al. 2007; Zhao et al. 2016).

Based on a new perspective established in recent years, MSCs can polarize into two different and identified phenotypes, MSC1 and MSC2 (depending on the specific TLRs stimulation) (Qu et al. 2018).

Based on this, the activation of TLR4 on MSCs has led to the formation of MSC1 phenotype; in this case, they show an inherently proinflammatory and anti-tumor activity (Waterman et al. 2010). However, stimulation of TLR3 on MSCs leads to the formation of MSC2 phenotype, which has anti-inflammatory properties and stimulates tumor growth and development (Bernardo and Fibbe 2013). Experimental evidence suggests that MSC1 multicellularity and different types of cancer cell lines reduce colony-forming units derived from tumor cells. Also, the created cultivation reduces the invasive power of tumor cells in spherical three-dimensional culture. Conversely, conflicting results were obtained after using MSC2 phenotype in multicellular cell cultures (Bunnell et al. 2010).

Studies by Romieu-Mourez et al. have shown that separate stimulation of TLRs on MSCs derived from mouse tissues produces cytokines IL-8, IL-6, and IL-1 (Romieu-Mourez

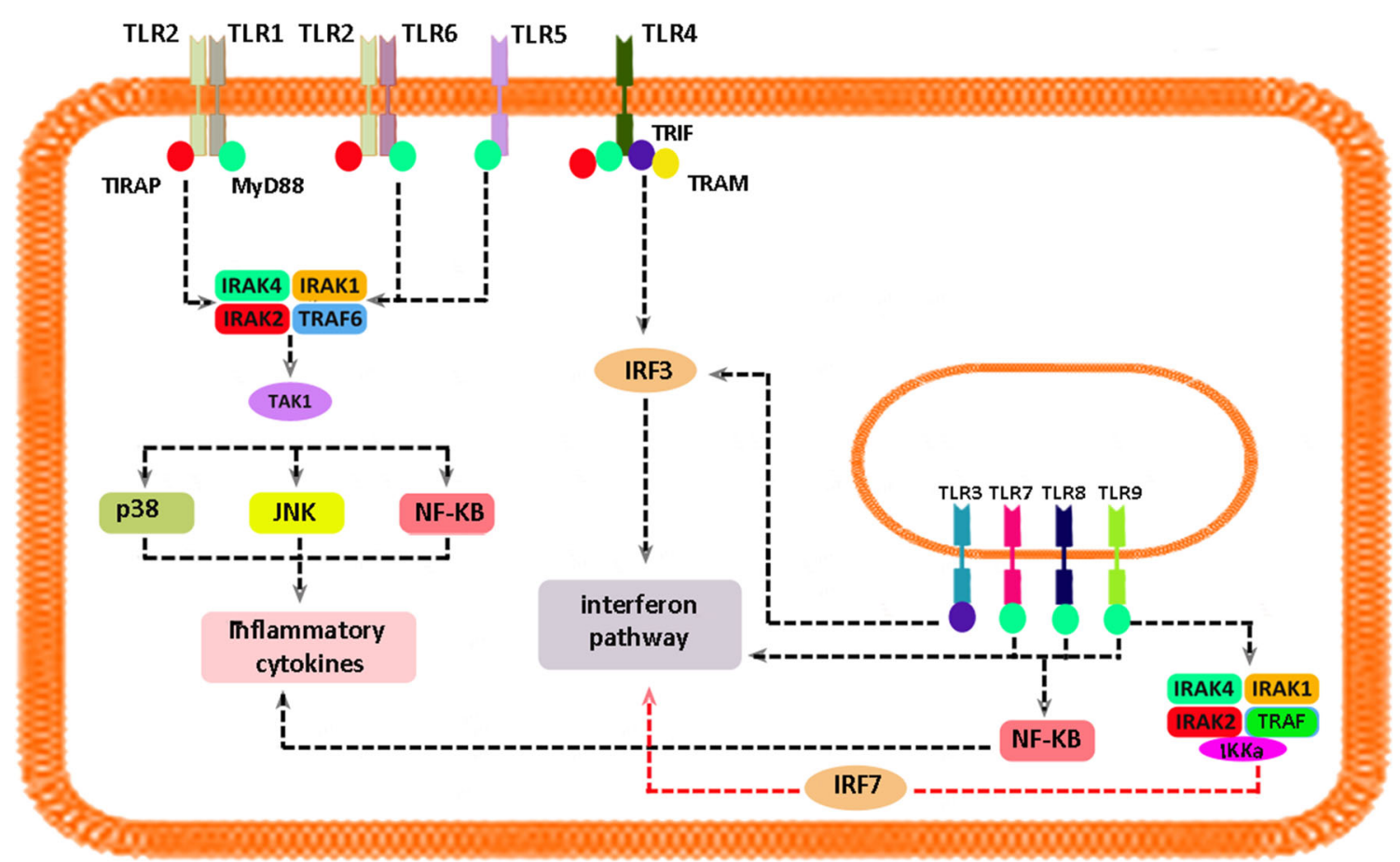

Fig. 1 Schematic location of TLRs in the cell and the signaling pathways associated with them 
et al. 2009). The group also stated that concomitant administration of MSCs (stimulated by IFN- $\gamma$ via TLRs and extracellular matrix components such as Matrigel matrix) led to local inflammatory production at the injection site, which could attract immune cells by this position. Overall, after artificial preactivation by using cytokines and TLR ligands, TLR activation in MSCs establishes an inflammatory site attracting different innate immune cells. Eventually, they regulate and modulate the attracted cells (Romieu-Mourez et al. 2009). This phenomenon may lead to extensive action and reactions in the position, including recall of neutrophils, secretion of different cytokine profiles by MSCs, and changes in their differentiating abilities (Romieu-Mourez et al. 2009; Bunnell et al. 2010; Waterman et al. 2012). Extensive studies are currently being conducted using the MSCs or MSC1 proinflammatory phenotype for therapeutic purposes against cancers. The results show that the first event in the development of neoplasms is the escape of tumor cells from the immune system and creating a localized tolerance at the tumor site. The escape of tumor cells from the immune system is vital for their growth and development. After MSC polarization to proinflammatory phenotype (MSC1), it is possible that this phenotype overcomes the escaping tumor cells from the immune system and creates conditions for the failure of the tolerance (which occurs primarily during tumor growth) (RomieuMourez et al. 2009; Bunnell et al. 2010; Waterman et al. 2012). The expression profile of TLRs and related ligands in MSCs isolated from umbilical cord blood is different from that of MSCs derived from bone marrow and can cause other effects of MSCs when faced with tumors (Berk et al. 2010). TLR4 and 5 signaling in cord blood MSCs is low and prompts the upregulation of a decreased number of proinflammatory cytokines (Berk et al. 2010).

Also, as mentioned earlier, the phenotype and function of MSCs can be modified by stimulating TLR4 and TLR3 and polarizing them to MSC1 and MSC2 phenotypes, respectively (Waterman et al. 2010). MSC1 releases inflammatory cytokines such as TGF- $\beta$, IL-8, and IL-6. Conversely, TLR3 stimulation induces MSC2 phenotype, which has an antiinflammatory function and stimulates tumor growth and development. MSC2 can release factors such as IDO, PGE2, IL4 , and IL-1R in the presence of tumors (Waterman et al. 2012). Besides, the polarization of MSCs to MSC1 phenotype with anti-tumor properties may lead to new strategies in treating cancers by MSCs to overcome these cells' adverse effects on tumors (Waterman et al. 2012). The findings confirm that MSCs with different tissue origins have different TLRs expression patterns (Shirjang et al. 2017). TLRs expression patterns can also be modified based on the tumor's microscopic environment's conditions and contents. For example, hypoxic conditions can affect adipose-derived MSCs by increasing the mRNA of TLR1, 2, 5, 9, and 10 (Lombardo and Delarosa 2010). Due to the dual and contradictory effects of
MSCs in their non-degraded form and the face of cancers, creating phenotypes with anti-tumor effects such as TLR4 stimulation and MSC1 phenotype may help to prevent the adverse effects of using these cells in cell therapy. This area of future research is likely to be one of this branch of treatment's main goals. In harmony with the polarization of MSCs and the existence of conditions and factors involved in directing these cells' dual effects, optimizing MSCs by providing sufficient conditions and factors in inducing anti-tumor effects of these cells is an attractive field for future research in this field. One of the strong candidates for immunotherapy is human MSCs (MSC), by their multivariate potential for immune system modulating features primarily depend on their origin, source, and niche. MSC may interact with their microenvironment by TLRs, and their ligands may help as a practical step in controlling biological function and distinguishing the diseases (Mekhemar et al. 2018). On the other hand, there are different signaling pathways for interaction between viral infection and these receptors and viral RNA are the significant components which are distinguished by TLRs (Yan et al. 2020). TLR7/8 are the main instigators that trigger the immune system after dealing with ssRNA virus and recruitment of MyD88 and IL-1R family, and release the NF- $\kappa \beta$, and also proinflammatory cytokines (Yan et al. 2020).

Until preparing this mini-review, 150 clinical trials using different sources of stem cells to treat severe COVID-19 patients have been registered in the clinical library of different countries (https://celltrials.org/cells-data). However, few clinical studies using MSCs treatment in COVID-19 patients display improved clinical signs of enrolled patients with no adverse effects. Moreover, a few clinical studies have been registered on clinicaltrals.gov for MSC-derived exosomes using COVID-19 treatment (Golchin 2020). Furthermore, there is a nonrandomized open-label cohort clinical study of commercial MSC-derived exosomes in COVID-19 patients, which has reported positive impacts of these exosomes on pulmonary inflammation COVID-19 patients (Sengupta et al. 2020).

\section{Conclusion}

Because efforts to control unusual viral infections via pharmacological treatments have so far been disappointing, cellbased therapy and especially MSC therapy are being studied based on MSC's stemness and their accessibility. Concurrently, severe acute respiratory syndrome coronavirus 2 (SARS-CoV-2) has accumulated global attention for causing the COVID-19 pandemic. Hence, some studies have focused on elevating their therapeutic potential for different conditions, wild-type viral infectious diseases. Given that there are complex signaling pathways for interaction between viral infection and TLRs, viral RNA is the main component 
distinguished by TLRs. There are a few therapeutic choices for treating COVID-19 patients; hence, targeting TLRs using pharmacological agents for clinical studies may present significant therapeutic approaches in the fight against COVID19. Given that the TLRs are immediately capable of detecting internal and external hazard signals, and their stimulation has an intense effect on the ability to proliferate, differentiate, migrate, and survival of MSCs, it seems stimulation of these receptors can have a primary effect on the interaction of MSCs and immune cells, developing the ability to modify the immune system activity. Therefore, MSC polarization presents a useful idea to perform these heterogeneous preparations of cells more stable and provides a critical aspect to study to develop current MSC-based therapies to treat viral diseases such as severe COVID-19. However, ongoing studies are aimed at promoting the potential of MSCs in future clinical applications.

Author contribution Ali Golchin and Shiva Gholizadeh conceptualized the outline and topic of the article. Ali Golchin finalized the manuscript. All authors participated in designing the study, drafting, writing, and editing the manuscript and approving it for submission.

\section{Declarations}

Conflict of interest The authors declare that they have no conflicts of interest.

\section{References}

Abdollahi-Roodsaz S, Joosten LAB, Koenders MI et al (2008) Stimulation of TLR2 and TLR4 differentially skews the balance of $\mathrm{T}$ cells in a mouse model of arthritis. $\mathrm{J}$ Clin Invest 118:205-216. https://doi.org/10.1172/JCI32639

Al-Anazi K, Al-Anazi WK, Al-Jasser A (2020) The rising role of mesenchymal stem cells in the treatment of various infectious complications. In: Update on mesenchymal and induced pluripotent stem cells. IntechOpen

Akira S, Sato S (2003) Toll-like receptors and their signaling mechanisms. Scand J Infect Dis 35:555-562. https://doi.org/10.1080/ 00365540310015683

Al-Anazi KA, Al-Anazi WK, Al-Jasser AM (2019) The beneficial effects of varicella zoster virus. J Hematol Clin Res 3:016-049. https://doi. org/10.29328/journal.jhcr.1001010

Berk LCJ van den, Jansen BJH, Siebers-Vermeulen KGC, et al (2010) Toll-like receptor triggering in cord blood mesenchymal stem cells. J Cell Mol Med 13:3415-3426. https://doi.org/10.1111/j.15824934.2008.00653.x

Bernardo ME, Fibbe WE (2013) Mesenchymal stromal cells: Sensors and switchers of inflammation. Cell Stem Cell 13:392-402

Bian P, Ye C, Zheng X et al (2017) Mesenchymal stem cells alleviate Japanese encephalitis virus-induced neuroinflammation and mortality. Stem Cell Res Ther:8. https://doi.org/10.1186/s13287-0170486-5

Bunnell BA, Betancourt AM, Sullivan DE (2010) New concepts on the immune modulation mediated by mesenchymal stem cells. Stem Cell Res. Ther: 1
Chan MCW, Kuok DIT, Leung CYH et al (2016) Human mesenchymal stromal cells reduce influenza A H5N1-associated acute lung injury in vitro and in vivo. Proc Natl Acad Sci U S A 113:3621-3626. https://doi.org/10.1073/pnas.1601911113

Chandra PK, Gerlach SL, Wu C et al (2018) Mesenchymal stem cells are attracted to latent HIV-1-infected cells and enable virus reactivation via a non-canonical PI3K-NFKB signaling pathway. Sci Rep 8. https://doi.org/10.1038/s41598-018-32657-y

Chen G-Y, Shiah H-C, Su H-J et al (2009) Baculovirus transduction of mesenchymal stem cells triggers the toll-like receptor 3 pathway. $\mathrm{J}$ Virol 83:10548-10556. https://doi.org/10.1128/jvi.01250-09

Chen J, Hu C, Chen LL et al (2020a) Clinical study of mesenchymal stem cell treatment for acute respiratory distress syndrome induced by epidemic influenza A (H7N9) infection: A hint for COVID-19 treatment. Engineering. https://doi.org/10.1016/j.eng.2020.02.006

Chen X, Shan Y, Wen Y et al (2020b) Mesenchymal stem cell therapy in severe COVID-19: A retrospective study of short-term treatment efficacy and side effects. J Infect. https://doi.org/10.1016/j.jinf. 2020.05.020

Cho HH, Shin KK, Kim YJ et al (2010) NF-KB activation stimulates osteogenic differentiation of mesenchymal stem cells derived from human adipose tissue by increasing TAZ expression. J Cell Physiol 223:168-177. https://doi.org/10.1002/jcp.22024

El Omar R, Beroud J, Stoltz JF et al (2014) Umbilical cord mesenchymal stem cells: The new gold standard for mesenchymal stem cell-based therapies? Tissue Eng. - Part B Rev 20:523-544

Fawzy-El-Sayed K, Mekhemar M, Adam-Klages S et al (2016) TLR expression profile of human gingival margin-derived stem progenitor cells. Med Oral Patol Oral Cir Bucal 21:e30-e38. https://doi.org/ $10.4317 /$ medoral.20593

Golchin A (2020) Cell-based therapy for severe COVID-19 patients: Clinical trials and cost-utility. Stem Cell Rev Reports:1-7. https:// doi.org/10.1007/s12015-020-10046-1

Golchin A, Hosseinzadeh S, Jouybar A et al (2020a) Wound healing improvement by curcumin-loaded electrospun nanofibers and BFP-MSCs as a bioactive dressing. Polym Adv Technol 31: pat.4881. https://doi.org/10.1002/pat.4881

Golchin A, Rekabgardan M, Taheri RA, Nourani MR (2018) Promotion of cell-based therapy: special focus on the cooperation of mesenchymal stem cell therapy and gene therapy for clinical trial studies. In: Turksen K (ed) Advances in experimental medicine and biology. Springer, New York, NY, pp 103-118

Golchin A, Seyedjafari E, Ardeshirylajimi A (2020b) Mesenchymal stem cell therapy for COVID-19: present or future. Stem Cell Rev. Reports 16:427-433

Golchin A, Shams F, Karami F (2020c) Advancing mesenchymal stem cell therapy with CRISPR/Cas9 for clinical trial studies. Springer

Goulopoulou S, McCarthy CG, Clinton Webb R (2016) Toll-like receptors in the vascular system: Sensing the dangers within. Pharmacol Rev 68:142-167. https://doi.org/10.1124/pr.114.010090

Heinz S, Haehnel V, Karaghiosoff M et al (2003) Species-specific regulation of toll-like receptor 3 genes in men and mice. J Biol Chem 278:21502-21509. https://doi.org/10.1074/jbc.M301476200

Hwa Cho H, Bae YC, Jung JS (2006) Role of toll-like receptors on human adipose-derived stromal cells. Stem Cells 24:2744-2752. https://doi. org/10.1634/stemcells.2006-0189

Judge AD, Sood V, Shaw JR et al (2005) Sequence-dependent stimulation of the mammalian innate immune response by synthetic siRNA. Nat Biotechnol 23:457-462. https://doi.org/10.1038/nbt1081

Khatri M, Richardson LA, Meulia T (2018) Mesenchymal stem cell-derived extracellular vesicles attenuate influenza virus-induced acute lung injury in a pig model. Stem Cell Res Ther:9. https://doi.org/10. 1186/s13287-018-0774-8

Khoury M, Cuenca J, Cruz FF et al (2020) Current status of cell-based therapies for respiratory virus infections: applicability to COVID19. Eur Respir J 55 
Leng Z, Zhu R, Hou W et al (2020) Transplantation of ACE2Mesenchymal stem cells improves the outcome of patients with covid-19 pneumonia. Aging Dis 11:216-228. https://doi.org/10. 14336/AD.2020.0228

Li C, Li B, Dong Z et al (2014) Lipopolysaccharide differentially affects the osteogenic differentiation of periodontal ligament stem cells and bone marrow mesenchymal stem cells through Toll-like receptor 4 mediated nuclear factor $\mathrm{kb}$ pathway. Stem Cell Res Ther 5. https:// doi.org/10.1186/scrt456

Li Y, Xu J, Shi W et al (2016a) Mesenchymal stromal cell treatment prevents H9N2 avian influenza virus-induced acute lung injury in mice. Stem Cell Res Ther 7:1-11. https://doi.org/10.1186/s13287016-0395-Z

Li YH, Xu Y, Wu HM et al (2016b) Umbilical cord-derived mesenchymal stem cell transplantation in hepatitis B virus related acute-onchronic liver failure treated with plasma exchange and entecavir: A 24-month prospective study. Stem Cell Rev Reports 12:645-653. https://doi.org/10.1007/s12015-016-9683-3

Lin BL, Chen JF, Qiu WH et al (2017) Allogeneic bone marrow-derived mesenchymal stromal cells for hepatitis B virus-related acute-onchronic liver failure: A randomized controlled trial. Hepatology 66: 209-219. https://doi.org/10.1002/hep.29189

Liotta F, Angeli R, Cosmi L et al (2008) Toll-like receptors 3 and 4 are expressed by human bone marrow-derived mesenchymal stem cells and can inhibit their T-Cell modulatory activity by impairing notch signaling. Stem Cells 26:279-289. https://doi.org/10.1634/ stemcells.2007-0454

Liu B, Ding F, Hu D et al (2018) Human umbilical cord mesenchymal stem cell conditioned medium attenuates renal fibrosis by reducing inflammation and epithelial-to-mesenchymal transition via the TLR4/NF-KB signaling pathway in vivo and in vitro. Stem Cell Res Ther 9:1-14. https://doi.org/10.1186/s13287-017-0760-6

Liu X, Wang C, Ye Z et al (2013) Higher expression of toll-like receptors 2, 3, 4, and 8 in ocular Behcet's disease. Investig Ophthalmol Vis Sci 54:6012-6017. https://doi.org/10.1167/iovs.13-12159

Lombardo E, Delarosa O (2010) Modulation of adult mesenchymal stem cells activity by toll-like receptors: Implications on therapeutic potential. Mediators Inflamm. 2010

Lombardo E, Delarosa O, Mancheño-Corvo P et al (2009) Toll-like receptor-mediated signaling in human adipose-derived stem cells: Implications for immunogenicity and immunosuppressive potential. Tissue Eng - Part A 15:1579-1589. https://doi.org/10.1089/ten.tea. 2008.0340

Maytawan Thanunchai SH, Thitithanyanont A (2015) Mesenchymal stromal cells and viral infection. Stem Cells Int 8

Mekhemar MK, Dörfer CE, El-Sayed KMF (2018) Toll-like receptors: The key of immunotherapy in MSCs. In: Immunoregulatory Aspects of Immunotherapy. InTech

Mestas J, Hughes CCW (2004) Of mice and not men: differences between mouse and human immunology. J Immunol 172:2731-2738. https://doi.org/10.4049/jimmunol.172.5.2731

Monguió-Tortajada M, Bayes-Genis A, Rosell A, Roura S (2020) Are mesenchymal stem cells and derived extracellular vesicles valuable to halt the COVID-19 inflammatory cascade? Current evidence and future perspectives:Thorax thoraxjnl-2020-215717. https://doi.org/ 10.1136/thoraxjnl-2020-215717

Najar M, Krayem M, Meuleman N et al (2017) Mesenchymal stromal cells and toll-like receptor priming: A critical review. Immune Netw. 17:89-102

Nemeth K, Mayer B, Mezey E (2010) Modulation of bone marrow stromal cell functions in infectious diseases by toll-like receptor ligands. J. Mol. Med. 88:5-10

Nicola MD, Di Carlo-Stella C, Magni M et al (2002) Human bone marrow stromal cells suppress T-lymphocyte proliferation induced by cellular or nonspecific mitogenic stimuli. Blood 99:3838-3843. https://doi.org/10.1182/blood.V99.10.3838
Pevsner-Fischer M, Morad V, Cohen-Sfady M et al (2007) Toll-like receptors and their ligands control mesenchymal stem cell functions. Blood 109:1422-1432. https://doi.org/10.1182/blood-2006-06028704

Qian X, Xu C, Fang S et al (2016) Exosomal MicroRNAs derived from umbilical mesenchymal stem cells inhibit hepatitis $\mathrm{C}$ virus infection. Stem Cells Transl Med 5:1190-1203. https://doi.org/10.5966/sctm. 2015-0348

Qu G, Xie X, Li X et al (2018) Immunomodulatory function of mesenchymal stem cells: regulation and application. J Cell Immunother 4: 1-3. https://doi.org/10.1016/j.jocit.2018.09.001

Raicevic G, Najar M, Stamatopoulos B et al (2011) The source of human mesenchymal stromal cells influences their TLR profile as well as their functional properties. Cell Immunol 270:207-216. https://doi. org/10.1016/j.cellimm.2011.05.010

Rizzo R, Campioni D, Stignani M et al (2008) A functional role for soluble HLA-G antigens in immune modulation mediated by mesenchymal stromal cells. Cytotherapy 10:364-375. https://doi.org/ 10.1080/14653240802105299

Rogier R, Koenders MI, Abdollahi-Roodsaz S (2015) Toll-like receptor mediated modulation of $\mathrm{T}$ cell response by commensal intestinal microbiota as a trigger for autoimmune arthritis. J. Immunol. Res. 2015

Romieu-Mourez R, François M, Boivin M-N et al (2009) Cytokine modulation of TLR expression and activation in mesenchymal stromal cells leads to a proinflammatory phenotype. J Immunol 182:79637973. https://doi.org/10.4049/jimmunol.0803864

Dela RO, Dalemans W, Lombardo E (2012) Toll-like receptors as modulators of mesenchymal stem cells. Front. Immunol. 3:182

Sánchez-Guijo F, García-Arranz M, López-Parra M et al (2020) Adiposederived mesenchymal stromal cells for the treatment of patients with severe SARS-CoV-2 pneumonia requiring mechanical ventilation. A proof of concept study. EClinicalMedicine. https://doi.org/10. 1016/j.eclinm.2020.100454

Sengupta V, Sengupta S, Lazo A et al (2020) Exosomes derived from bone marrow mesenchymal stem cells as treatment for severe COVID-19. Stem Cells Dev 29:747-754. https://doi.org/10.1089/ scd. 2020.0080

Shi M, Zhang Z, Xu R et al (2012) Human mesenchymal stem cell transfusion is safe and improves liver function in acute-on-chronic liver failure patients. Stem Cells Transl Med 1:725-731. https://doi. org/10.5966/sctm.2012-0034

Shirjang S, Mansoori B, Solali S et al (2017) Toll-like receptors as a key regulator of mesenchymal stem cell function: An up-to-date review. Cell Immunol 315:1-10

Tomchuck SL, Zwezdaryk KJ, Coffelt SB et al (2008) Toll-like receptors on human mesenchymal stem cells drive their migration and immunomodulating responses. Stem Cells 26:99-107. https://doi. org/10.1634/stemcells.2007-0563

Tomic S, Djokic J, Vasilijic S et al (2011) Immunomodulatory properties of mesenchymal stem cells derived from dental pulp and dental follicle are susceptible to activation by toll-like receptor agonists. Stem Cells Dev 20:695-708. https://doi.org/10.1089/scd.2010.0145

Wakatabe M, Ishii M, Namkoong H et al (2012) Clarithromycin modulates cytokine expression profiles. In: Murine macrophages and dendritic cells during influenza a virus infection. American Thoracic Society 2012 International Conference, San Francisco, California, pp A5718-A5718

Waterman RS, Henkle SL, Betancourt AM (2012) Mesenchymal stem cell 1 (MSC1)-based therapy attenuates tumor growth whereas MSC2-treatment promotes tumor growth and metastasis. PLoS One 7. https://doi.org/10.1371/journal.pone.0045590

Waterman RS, Tomchuck SL, Henkle SL, Betancourt AM (2010) A new mesenchymal stem cell (MSC) paradigm: Polarization into a proinflammatory MSC1 or an immunosuppressive MSC2 phenotype. PLoS One 5:e10088. https://doi.org/10.1371/journal.pone.0010088 
West AP, Koblansky AA, Ghosh S (2006) Recognition and signaling by toll-like receptors. Annu Rev Cell Dev Biol 22:409-437

Xu X, Wang W, Lin L, Chen P (2020) Liraglutide in combination with human umbilical cord mesenchymal stem cell could improve liver lesions by modulating TLR4/NF-kB inflammatory pathway and oxidative stress in T2DM/NAFLD rats. Tissue Cell 66:101382. https:// doi.org/10.1016/j.tice.2020.101382

Yan E, Jiang J, Yang X et al (2020) pH-sensitive core-shell electrospun nanofibers based on polyvinyl alcohol/polycaprolactone as a potential drug delivery system for the chemotherapy against cervical cancer. J Drug Deliv Sci Technol 55:101455. https://doi.org/10.1016/j. jddst.2019.101455

Zhang Y, Ding J, Ren S et al (2020) Intravenous infusion of human umbilical cord Wharton's jelly-derived mesenchymal stem cells as a potential treatment for patients with COVID-19 pneumonia. Stem Cell Res Ther 11. https://doi.org/10.1186/s13287-020-01725-4

Zhang Z, Fu J, Xu X et al (2013) Safety and immunological responses to human mesenchymal stem cell therapy in difficult-to-treat HIV-1infected patients. AIDS 27:1283-1293. https://doi.org/10.1097/ QAD.0b013e32835fab77

Zhao Q, Ren H, Han Z (2016) Mesenchymal stem cells: Immunomodulatory capability and clinical potential in immune diseases. J Cell Immunother 2:3-20

Publisher's note Springer Nature remains neutral with regard to jurisdictional claims in published maps and institutional affiliations. 